\title{
Role of the p53-TRPM1/miR-211-MMP9 axis in UVB-induced human melanocyte migration and its potential in repigmentation
}

\author{
MENGYUN SU, FANG MIAO, SHAN JIANG, YING SHI, LONGFEI LUO, \\ XIAOLEI HE, JING WAN, SHIZHENG XU and TIE-CHI LEI
}

Department of Dermatology, Renmin Hospital of Wuhan University, Wuhan, Hubei 430060, P.R. China

Received September 19, 2019; Accepted December 16, 2019

DOI: $10.3892 / \mathrm{ijmm} .2020 .4478$

\begin{abstract}
Clinical studies have proven that ultraviolet B (UVB) based phototherapy can induce perifollicular and marginal repigmentation patterns in the skin of vitiligo patients. It is, however, difficult to conceive how melanocytes can easily exit from their tightly interconnected epidermal microenvironment to re-enter a different location in the skin to establish a new network with neighboring keratinocytes. While it is known that matrix metalloprotease 9 (MMP9) is involved in the degradation of the extracellular matrix in physiological or pathological processes, little is known about whether MMP9 affects melanocyte migration in vitiligo repigmentation. To investigate the effects of the $\mathrm{p} 53$ - transient receptor potential cation channel subfamily $\mathrm{M}$ member 1 (TRPM1)/microRNA (miR/miRNA)-211-MMP9 axis to regulate melanocyte migration following exposure to UVB, the expression profile of MMP9 in cultured human melanocytes transfected with or without the miR-211-mimic and p53-GFP lentiviral vector, respectively were determined. Quantitative polymerase chain reaction and western blotting were used to examine p53, TRPM1 and MMP9 mRNA and protein levels in UVB-exposed and unexposed cells. The capacity of melanocytes to migrate on collagen IV substrate was estimated using a Transwell migration assay. Interestingly, the upregulation of p53 and MMP9 at the mRNA and protein levels was evident in melanocytes treated with single or repeat exposures to UVB, whereas levels of TRPM1 and miR-211 were significantly suppressed in UVB-exposed melanocytes compared with the UVB-unexposed control cells. These results indicate that the p53-TRPM1/miR-211-MMP9 axis is significantly activated in melanocytes exposed to UVB. Notably, the ability of melanocyte migration was altered by the overexpression of p53 using a lentiviral vector and by the upregulation of miR-211 using
\end{abstract}

Correspondence to: Dr Tie-Chi Lei, Department of Dermatology, Renmin Hospital of Wuhan University, 238 Jiefang Road, Wuchang, Wuhan, Hubei 430060, P.R. China

E-mail: tchlei@whu.edu.cn

Key words: melanocyte, migration; p53, microRNA-211, ultraviolet B an miRNA mimic. That altered migration could be neutralized by co-treatment with GM6001 (a broad-spectrum MMP inhibitor). Overall, these results show that the MMP9-mediated migration of melanocytes is regulated by a novel mechanism driven by the p53-TRPM1/miR-211-MMP9 axis. Activation of the $\mathrm{p53-TRPM1/miR-211-MMP9}$ axis potentially represents an attractive therapeutic target to improve repigmentation outcomes in vitiligo patients.

\section{Introduction}

Vitiligo is the most common depigmentary disorder of the skin, affecting $0.5-2 \%$ of the population worldwide. Vitiligo is predominantly characterized by white patches of the skin, due to the chronic and progressive loss of melanocytes from the epidermis and the follicular reservoir, which may cause significant social and psychological impacts on patients, especially teenagers (1-3). Although autoimmune-mediated cell death is currently accepted as the leading cause for the disappearance of melanocytes in most cases of vitiligo, the molecular mechanism by which a melanocyte-specific immune response is exclusively triggered has never been clearly determined (4). Within the skin, mature melanocytes reside in the basal layer of the epidermis where they form epidermal melanin units comprised of tight anatomical and functional relationships between individual melanocytes and 30-40 neighboring keratinocytes (5). Unlike keratinocytes, which possess hemidesmosomes and desmosomes that anchor them to the underlying basement membrane and adjacent basal keratinocytes, epidermal melanocytes are simply anchored by the cell-matrix and/or cell-cell adhesion molecules such as integrin, E-cadherin and discoidin domain receptor $1(6,7)$. The reservoirs of melanocytes at the cellular and molecular levels after their selective disappearance from vitiliginous skin remain to be investigated. Vitiligo keratinocytes must re-establish cell-cell contacts after melanocytes disappear, speculatively forming a defective cell complex with an impaired ability to photoprotect the skin against damage from harmful ultraviolet (UV) radiation (8).

Current therapeutic modalities for vitiligo are aimed at arresting progression of the disease and stimulating skin repigmentation (a process that recruits existing melanocytes and their precursors or stem cells back to the depigmented skin lesions) $(5,9)$. UVB-based phototherapy has been 
clinically proven to be efficient for inducing repigmentation $(5,10)$. Different skin repigmentation patterns, such as perifollicular, marginal, diffuse and combined patterns, have been observed in vitiligo patients who have undergone UVB-based phototherapy, suggesting that the replenishment of melanocytes probably comes from diverse sources in the epidermis and/or in hair follicles (11). Over the past decades, effort has been devoted to investigating whether dormant melanocyte stem cells that reside in the bulge region of hair follicles are awakened to participate in the perifollicular repigmentation of the skin $(5,12-14)$. In contrast, much less is understood about the induction of marginal repigmentation of the skin, which possibly relies on the activation and migration of melanocytes existing at the border of the depigmented vitiligo macules.

The migration of melanocytes released from the microenvironment of basal and suprabasal layers in pigmented human epidermis in response to UVB phototherapy raises intriguing questions. The critical issues that remain to be resolved include: i) Which cells are preferentially mobilized by UVB irradiation, ii) whether the migrating cells arise from a single population of immature and/or mature melanocytes, and iii) whether the migrating cells transiently secrete one or more proteolytic enzymes that enable melanocytes to move out by dissolving intercellular adhesive structures. Several studies have demonstrated that the expression of matrix metalloproteases (MMPs) in melanocytes appears to be correlated with tissue remodeling or replasticity (15-17), little is known about whether MMP9 affects melanocyte migration in vitiligo repigmentation. The present study provides unequivocal evidence that the p53-TRPM1/microRNA (miRNA/miR)-211-MMP9 axis is critical for melanocyte migration induced by UVB exposure, which has potential implications for understanding the molecular mechanisms underlying UVB-induced repigmentation in vitiligo.

\section{Materials and methods}

Cell culture and $U V B$ radiation. The primary human epidermal melanocytes were isolated from healthy juvenile foreskin tissues, a total of 60 specimens, collected after circumcision between September 2017 and May 2019 (donor age range, 13-19 years). After removing the subcutaneous tissue, the samples were cut into small pieces and incubated with $0.25 \%$ dispase at $4^{\circ} \mathrm{C}$ overnight to separate the epidermis and dermis (18). The present study was approved by the Ethical Committee of the Renmin Hospital of Wuhan University. Written informed consent was obtained from each participant before enrollment. Melanocytes were cultured with complete Medium 254 supplemented with Human Melanocyte Growth Supplement and $100 \mathrm{U} / \mathrm{ml}$ penicillin/streptomycin (all from Cascade Biologics) in a humidified atmosphere at $37^{\circ} \mathrm{C}$ and $5 \% \mathrm{CO}_{2}$. Cells used in the present experiments were from passages 2 to 6 . Cells were seeded in 6 -well plates at a population density of $5 \times 10^{5}$. Before irradiation, the medium was removed and warm PBS was used to wash the cells. The cells were treated with single or repeated exposures to UVB using a high dose targeted phototherapy system (The Daavlin Company) with a maximum wavelength at $311 \mathrm{~nm}$. The cumulative dose of UVB irradiation is indicated in the figure legends. Cells of the UVB-unexposed control were treated in same way but without UVB exposure.

Semi-quantitative reverse transcription (RT)-PCR and quantitative PCR ( $q P C R)$. Total RNAs were purified using TRIzol reagent (Invitrogen; Thermo Fisher Scientific, Inc.) according to the protocol of the manufacturer. cDNAs were synthesized from total RNAs $(1.5 \mu \mathrm{g})$ using a Moloney murine leukemia virus reverse transcriptase first strand kit (Invitrogen; Thermo Fisher Scientific, Inc.). RT-PCR was performed under the following cycle parameters: Predenaturation at $95^{\circ} \mathrm{C}$ for $2 \mathrm{~min}$, followed by $35 \mathrm{cycles}$ of $95^{\circ} \mathrm{C}$ for $30 \mathrm{sec}, 55^{\circ} \mathrm{C}$ for $30 \mathrm{sec}$ and $72^{\circ} \mathrm{C}$ for $30 \mathrm{sec}$. PCR was performed in triplicate with SYBR-Green PCR core reagents (Applied Biosystems; Thermo Fisher Scientific, Inc.), $50 \mathrm{ng}$ cDNA and $1 \mu \mathrm{M}$ forward and reverse primers for the following human genes: p53 (forward: 5'-GTCTACCTCCCGCCATAA-3'; reverse: 5'-GCA AGCAAGGGTTCAAAG-3'), TRPM1 (forward: 5'-TACACG CTATTTCCCCGATGA-3'; reverse: 5'-GCGCGTGATCTT TTGAACTTG-3'), MMP9 (forward: 5'-GCTACCACCTCG AACTTTGAC-3'; reverse: 5'-TCAGTGAAGCGGTACATA GGG-3') and the housekeeping gene $\beta$-actin (ACTB; forward: 5'-AGCGAGCATCCCCCAAAGTT-3'; reverse: 5'-GGGCAC GAAGGCTCATCATT-3') was used as an endogenous internal control for the normalization of p53, TRPM1, and MMP9 mRNA expression. For qPCR analysis of miR-211, $10 \mathrm{ng}$ total RNA was used in a TaqMan miRNA assay according to the manufacturer's protocol. Analysis of miRNA was carried out using a CFX96 Detection System (Bio-Rad Laboratories, Inc.) in triplicate and the SYBR-Green Supermix. miR-211 specific primers: (Forward: 5'-TGCGCTTCCCTTTGT CATCCTT-3'; reverse: 5'-CTCAAGTGTCGTGGAGTCGG CAA-3') loop primer: 5'-GTCGTATCCAGTGCAGGGTCC GAGGTATTCGCACTGGATACGACAGGCGAAG-3'). The expression of miR-211 was normalized to the U6 small nuclear RNA (forward: 5'-CTCGCTTCGGCAGCACAT-3'; reverse: 5'-AACGCTTCACGAATTTGCGT-3'). All primers used in this study were synthesized and supplied by Invitrogen; Thermo Fisher Scientific, Inc. qPCR was performed using an ABI 7500 system with the following cycle parameters: Denaturation at $95^{\circ} \mathrm{C}$ for $30 \mathrm{sec}$, followed by 40 cycles of $95^{\circ} \mathrm{C}$ for $5 \mathrm{sec}, 60^{\circ} \mathrm{C}$ for $4 \mathrm{sec}$ and $72^{\circ} \mathrm{C}$ for $30 \mathrm{sec}$. The purity of each PCR product was checked by dissociation curve analysis, as well as by running each sample on $1 \%$ agarose gels with Goldview (cat. no. 110214; SBSGene). Fold-change values were calculated using the formula of $2^{-\Delta \Delta \mathrm{Cq}}(19)$.

Western blotting analysis. Cells were harvested, washed in PBS and lysed in extraction buffer containing 1\% Nonidet P-40, 0.01\% SDS and a protease inhibitor cocktail (Roche Diagnostics). Protein contents were determined using a bicinchoninic acid assay kit (Pierce; Thermo Fisher Scientific, Inc.). Equal amounts of each protein extract ( $20 \mu \mathrm{g}$ per lane) were resolved using 10\% SDS-PAGE. Following transblotting onto Immobilon-P membranes (EMD Millipore) and blocking with 5\% nonfat milk in saline buffer for $1 \mathrm{~h}$ at room temperature, the membranes were incubated with antibodies to p53 (cat. no. ab179477; Abcam; 1:1,000), TRPM1 (cat. no. ab72154; Abcam; 1:1,500), MMP9 (cat. no. ab76003; Abcam; 1:1,000) and GAPDH (cat. no. ab37168; Abcam; 
1:10,000) for $1 \mathrm{~h}$ at room temperature. The membranes were then washed and incubated with HRP-conjugated goat anti-rabbit IgG (AS1107; Aspen Biological; 1:10,000) for $1 \mathrm{~h}$ at room temperature. Each membrane was then washed again and specific immunoreactive bands were visualized using an enhanced chemiluminescent reaction (ECL kit; Amersham; GE Healthcare). Signal intensities were quantified using ImageJ software version 1.47 (National Institutes of Health) and were normalized to GAPDH. All data were obtained from more than two independent experiments carried out in triplicate.

Immunofluorescent assay. Primary melanocytes $\left(5 \times 10^{5}\right)$ were seeded in 6-well culture plates containing coverslips. After attachment, the cells were treated with multiple UVB exposures for a cumulative dose of $70 \mathrm{~mJ} / \mathrm{cm}^{2}\left(8.75 \mathrm{~mJ} / \mathrm{cm}^{2}\right.$ per exposure in $5 \mathrm{~h}$ intervals for a total of 8 times). Subsequently, the cells were immediately fixed in $4 \%$ paraformaldehyde in PBS for $30 \mathrm{~min}$ at room temperature, permeabilized with $0.3 \%$ Triton $\mathrm{X}-100$ in PBS for $15 \mathrm{~min}$ and then blocked for $1 \mathrm{~h}$ at $37^{\circ} \mathrm{C}$ using a blocking buffer containing $10 \%$ normal goat serum (cat. no. C0265; Beyotime Institute of Biotechnology). The anti-MMP9 (cat. no. ab76003; Abcam; 1:200) antibody was diluted in blocking buffer and placed on the cells at $4^{\circ} \mathrm{C}$ overnight. After the incubation, cells on coverslips were washed three times in PBS and then incubated with goat anti-rabbit IgG (AS1109; Aspen Biological; 1:50) for $1 \mathrm{~h}$ at $37^{\circ} \mathrm{C}$. Nuclei were stained using $4^{\prime} 6^{\prime}$-diamidino-2-phenylindole (DAPI) solution for $10 \mathrm{~min}$ at room temperature. Imaging was performed using an FV1200 (Olympus Corporation) confocal microscope.

Cell migration assay. Cell migration was assessed using Transwell cell culture chambers as previously described (20). Polyvinylpyrrolidone-free polycarbonate filters with an $8.0-\mu \mathrm{m}$ pore size were pre-coated with $10 \mathrm{mg} / \mathrm{ml}$ collagen IV (C6745; Sigma-Aldrich; Merck KGaA) and placed on the lower surface of Transwell chambers (Costar, 3422; Corning, Inc.). The filters were dried overnight at room temperature, washed extensively in PBS and then dried immediately before use. Melanocytes $\left(1 \times 10^{5}\right)$ were seeded into the upper chambers, while the lower chambers were filled with fresh complete medium supplemented with or without 0.2 nM GM6001 (HY-15768; MedChemExpress). Cell culture inserts were treated with repeated exposure to UVB as detailed above. A total of $48 \mathrm{~h}$ later, the filters were fixed in methanol for $15 \mathrm{~min}$ at room temperature and stained with crystal violet for $20 \mathrm{~min}$ at room temperature. Cells that had migrated into the lower surface of the membrane were counted in five microscopic fields at x200 magnification using an automatic microscope (Olympus Corporation) at least three independent experiments.

miRNA transfection. Primary melanocytes were seeded in 6 -well culture plates at a population density of $5 \times 10^{5}$. miR-211-mimic and miR-negative control (miR-NC; cat. no. miR01101) were purchased from Guangzhou RiboBio Co., Ltd. After $24 \mathrm{~h}$ of culture, the transfection of $50 \mathrm{nM}$ miR-211-mimic (cat. no. miR10022694; sequence, 5'-GCA GGGACAGCAAAGGGGUGC-3') was performed with RiboFECT ${ }^{\mathrm{TM}} \mathrm{CP}$ transfection reagent (Guangzhou RiboBio
Co., Ltd.) according to the manufacturer's protocol. miR-NC, which contains a sequence that lacks homology with other miRNAs, was transfected under identical conditions into melanocytes as the NC. After $48 \mathrm{~h}$ of transfection, the cells were harvested and assayed using qPCR and western blotting.

Recombinant lentiviral vector transfection. In order to produce a p53 expression construct, the protein coding sequence region of the human p53 gene was synthesized and inserted into Bam H I/Age I restriction sites of the lentiviral expression vector GV358 that contains the EGFP gene (Shanghai GeneChem Co., Ltd.). 293T cells (Shanghai GeneChem Co., Ltd.) were transfected with a mixture of plasmids, including viral packaging plasmids and the p53 expression plasmid (GV359-p53) or the control plasmid (GV358) via Lipofectamine 2000 (Invitrogen; Thermo Fisher Scientific, Inc.) based on the manufacturer's protocol. The viral supernatant was collected at $48 \mathrm{~h}$ after transfection and was used to infect melanocytes. Evaluation of p53 expression was conducted using fluorescence microscopy at $48 \mathrm{~h}$ after viral infection by harvesting the cells and characterizing their levels of mRNA and protein expression by qPCR and western blotting, respectively.

Statistical analysis. Data are expressed as the mean \pm standard deviation from three independent experiments. SPSS version 19.0 (IBM Corps.) and GraphPad Prism 8 (GraphPad Software, Inc.) software were used for analysis of all data. Statistical differences were determined by Student's t-test or one-way analysis of variance with Tukey's post hoc test. $\mathrm{P}<0.05$ was considered to indicate a statistically significant difference.

\section{Results}

Activation of the p53-TRPM1/miR-211-MMP9 axis in melanocytes treated with single or repeated exposures to $U V B$. First, the expression profiles of the p53-TRPM1/miR-211-MMP9 axis in melanocytes treated with single or repeated exposures to UVB was examined. For the single UVB exposures, cultured human melanocytes were exposed to a single dose of UVB $\left(0,8.75,17.5,35\right.$ and $\left.70 \mathrm{~mJ} / \mathrm{cm}^{2}\right)$. After $8 \mathrm{~h}$, the mRNA and protein expression levels of p53, TRPM1 and MMP9 were measured using RT-qPCR and western blotting, respectively. The results (Fig. 1) showed that the expression levels of p53 and MMP9 mRNAs and proteins were upregulated in a UVB dose-dependent manner $\left(8.75-70 \mathrm{~mJ} / \mathrm{cm}^{2}\right)$. Interestingly, TRPM1 mRNA and protein levels were increased at lower doses $\left(8.75\right.$ and $\left.17.5 \mathrm{~mJ} / \mathrm{cm}^{2}\right)$ but were reduced at higher doses (35 and $70 \mathrm{~mJ} / \mathrm{cm}^{2}$ ) of UVB. To simulate clinical settings with multiple exposures to UVB, the cells were treated with repeated small UVB exposures for cumulative doses of 35 or $70 \mathrm{~mJ} / \mathrm{cm}^{2}\left(8.75 \mathrm{~mJ} / \mathrm{cm}^{2}\right.$ per exposure in $5 \mathrm{~h}$ intervals for a total of 4 or 8 times), as shown in Fig. 2. The upregulation of p53 and MMP9 mRNA and protein levels was seen in melanocytes treated with 4 or 8 exposures to UVB, whereas mRNA and protein levels of TRPM1 were significantly decreased in cells repeatedly exposed to UVB compared with the UVB-unexposed control cells. These results demonstrated that the p53-TRPM1/miR-211-MMP9 axis is significantly activated in melanocytes by single and by repeated exposures to UVB. 

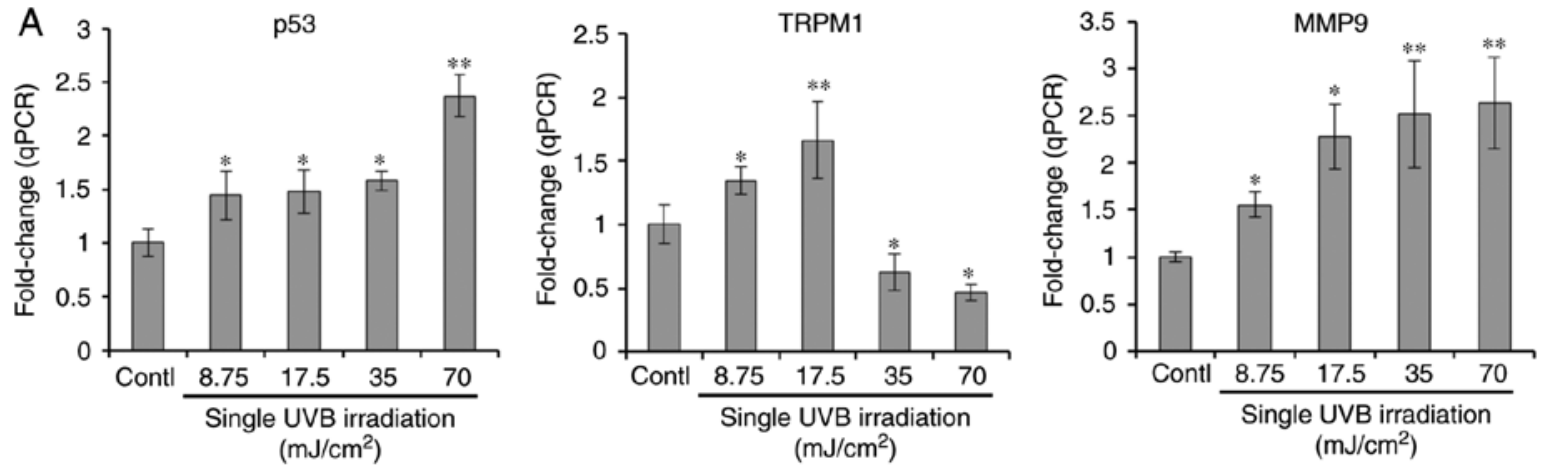

B
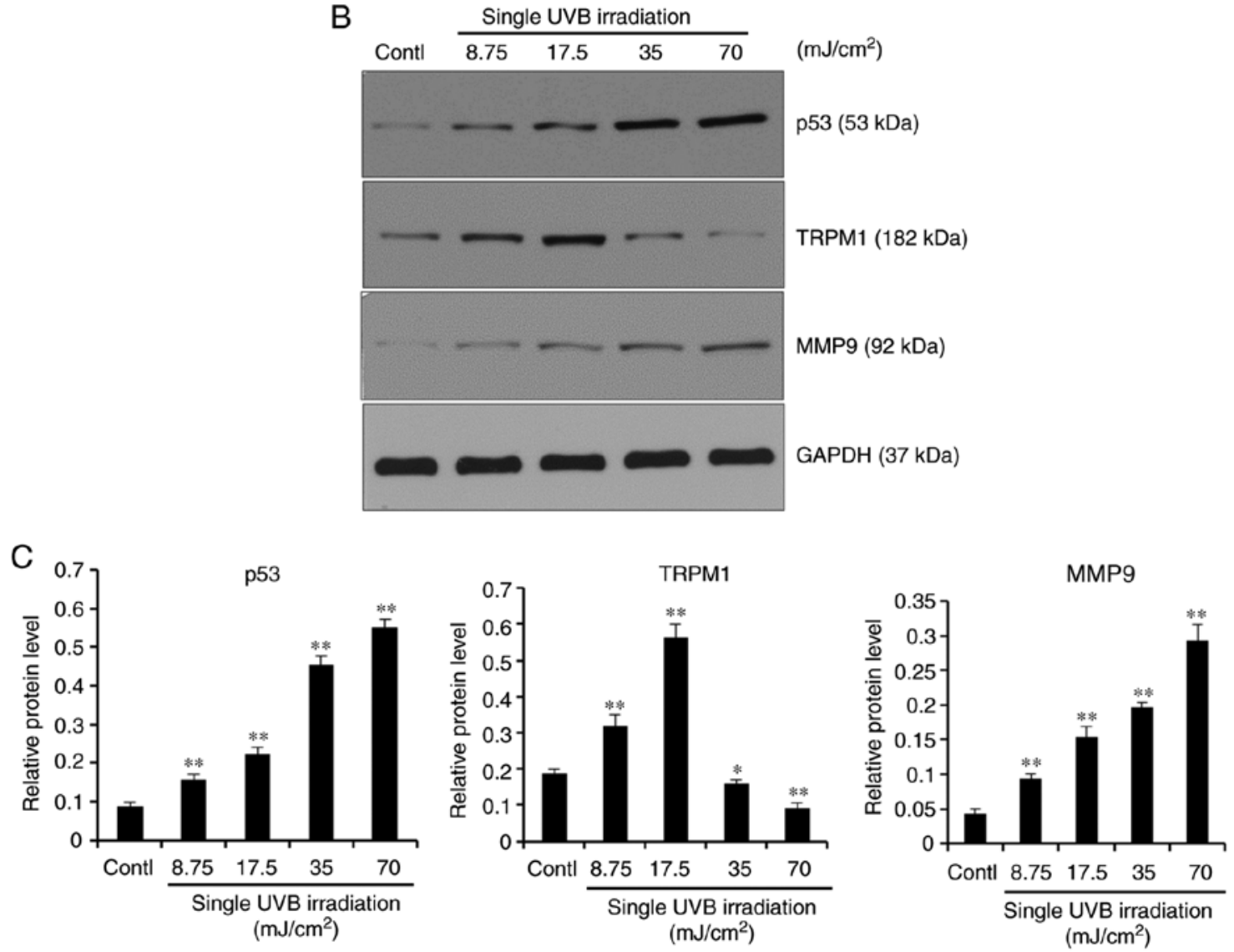

Figure 1. Expression profiles of the p53-TRPM1/miR-211-MMP9 axis in melanocytes treated with a single exposure to UVB. (A) The mRNA expression levels of p53, TRPM1 and MMP9 in melanocytes exposed to UVB or the control were detected by qPCR, and gene expression signals were normalized to the ACTB housekeeping gene. Data represent the mean \pm standard deviation of three independent experiments. (B) Cells were exposed to UVB at the indicated doses and were harvested $8 \mathrm{~h}$ later. Representative blots are shown of three independent blots with similar results. (C) Histograms showing the densitometric quantification of western blotting data with the mean \pm standard deviation of three independent experiments. Statistical analysis was performed using a one-way analysis of variance and compared with UVB-unexposed controls. " $\mathrm{P}<0.05$ and $^{* * *} \mathrm{P}<0.01$ vs. contl. miR, microRNA; UVB, ultraviolet B; ACTB, $\beta$-actin; qPCR, quantitative PCR; MMP, matrix metalloproteinase; TRPM1, p53-transient receptor potential cation channel subfamily M member 1; contl, control.

UVB-induced migration of melanocytes involves the downregulation of MMP9 by $m i R-211$. To investigate whether miR-211 is involved in the regulation of MMP9 and if this plays a central role in the regulation of melanocyte migration, the capacity of UVB-exposed melanocytes to migrate on collagen IV substrate was determined. The results (Fig. 3) showed that repeated UVB exposure significantly stimulated melanocyte migration and that there was a highly significant inverse association between miR-211 expression and MMP9 protein levels in the cells following repeated exposure to UVB. The mRNA expression of miR-211 was decreased and
MMP9 was upregulated. To further characterize the role of MMP9 in melanocyte migration, GM6001, a broad-spectrum MMP inhibitor, was used to block MMP9-mediated cell migration. As expected, the number of migrating cells in Transwell inserts with collagen IV-coated membranes was much lower in the presence of $0.2 \mathrm{nM}$ GM6001 compared with in the untreated control and even following exposure to UVB (Fig. 3C and D). Indeed, these findings strongly suggested that the post-transcriptional regulation of the MMP9 gene by miR-211 directly affects melanocyte migration. Furthermore, to determine whether miR-211 has an 

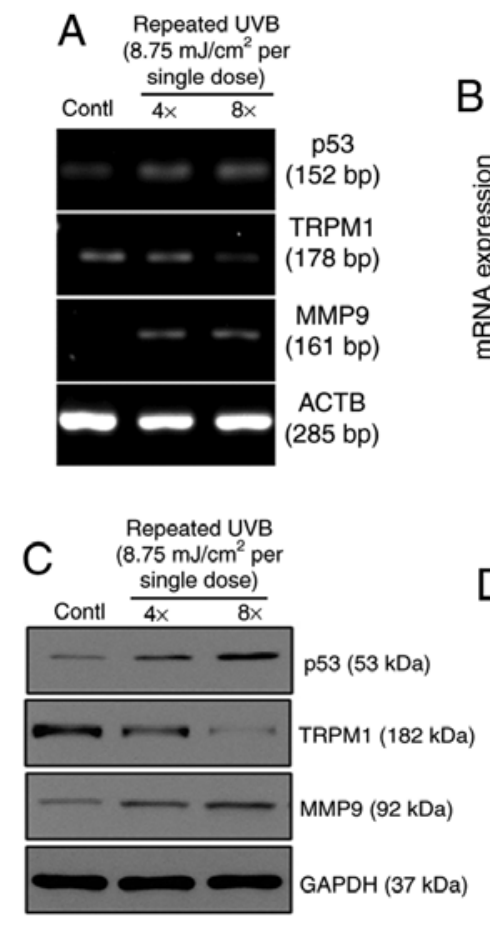
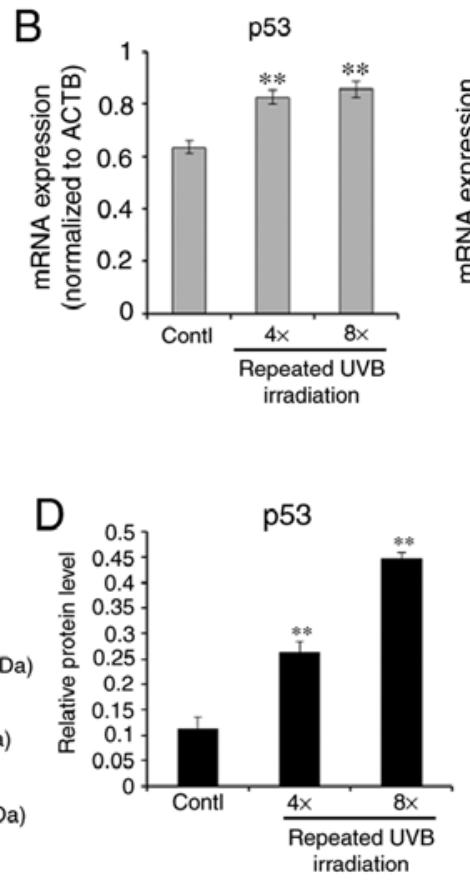
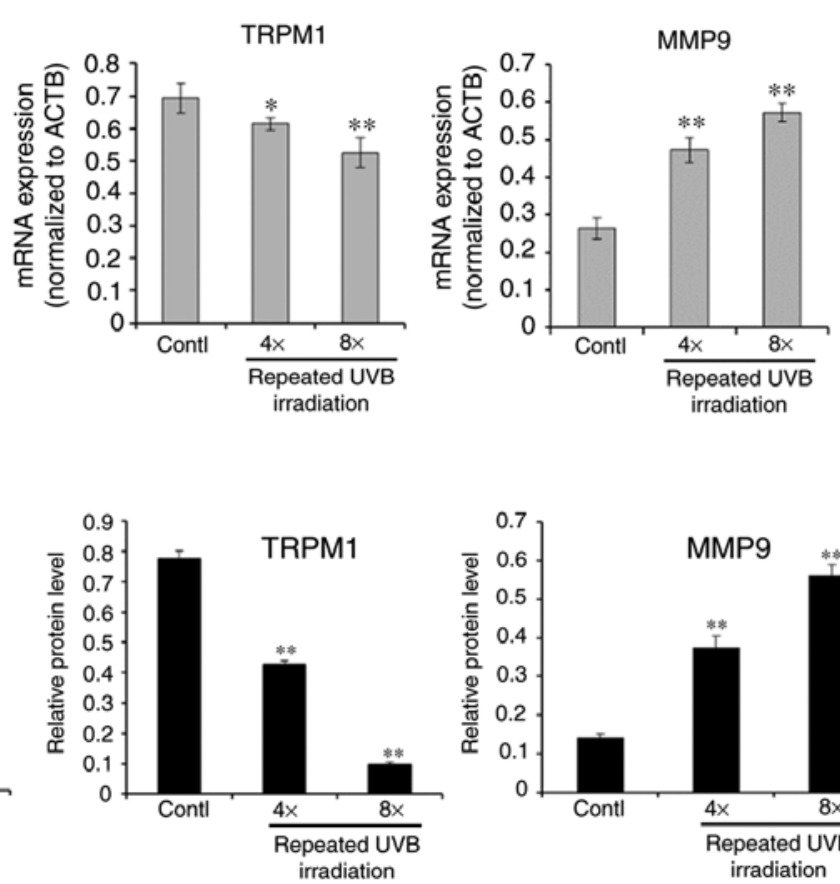

Figure 2. Expression profiles of the p53-TRPM1/miR-211-MMP9 axis in melanocytes treated with repeated exposures to UVB. (A) Melanocytes were treated with repeated UVB exposures for cumulative doses of $35(4 \mathrm{X})$ or $70(8 \mathrm{X}) \mathrm{mJ} / \mathrm{cm}^{2}$, given at $8.75 \mathrm{~mJ} / \mathrm{cm}^{2}$ per exposure in $5 \mathrm{~h}$ intervals for a total of 4 or 8 times. Semi-quantitative reverse transcription-PCR was performed to measure the expression levels of p53, TRPM1 and MMP9 mRNAs in melanocytes treated with repeated UVB exposures. Representative images are shown. (B) The relative expression levels of p53, TRPM1 and MMP9 mRNAs were normalized to the housekeeping gene $\beta$-actin and are reported as expression relative to three independent experiments. (C) Melanocytes were repeatedly exposed to UVB at the indicated doses and harvested after $8 \mathrm{~h}$ post-irradiation. Representative blots are shown among three independent blots with similar results. (D) Histograms showing the densitometric quantification of data with the mean \pm standard deviation of three independent experiments. ${ }^{*} \mathrm{P}<0.05$ and ${ }^{* *} \mathrm{P}<0.01$ vs. contl. $\mathrm{UVB}$, ultraviolet B; ACTB, $\beta$-actin; qPCR, quantitative PCR; MMP, matrix metalloproteinase; TRPM1, p53-transient receptor potential cation channel subfamily M member 1 .

effect on MMP9 expression and/or melanocyte migration, a chemically synthesized miRNA mimic was transiently transfected into melanocytes to determine the effects of miR-211. After transfection with the miR-211 mimic, the level of miR-211 was significantly increased in cells transfected with the miR-211 mimic compared with the miR-NC (Fig. 4A). Next, qPCR and western blotting were performed to assess the expression of MMP9 and found that the mRNA and protein levels of MMP9 were significantly suppressed after transfection with the miR-211 mimic (Fig. 4A and B). The Transwell assays showed that melanocyte migration was significantly reduced in the miR-211 mimic transfected cells compared with the miR-NC transfected cells (Fig. 4C). Likewise, the inhibition of the miR-211 mimic on melanocyte migration was neutralized by treatment with 0.2 nM GM6001 (Fig. 4C). Therefore, the present findings suggested that the post-transcriptional control of the MMP9 gene by miR-211 might play a critical role in the UVB-induced melanocyte migration.

$U V B$-induced melanocyte migration involves the upregulation of MMP9 by p53. In this study, the expression of endogenous p53 protein was induced by UVB irradiation (Figs. 1 and 2). To address the question of whether exogenous p53 overexpression would have a similar role in melanocyte migration, the effect of a lentiviral vector-mediated overexpression of p53 on the migration of cultured human melanocytes was examined. As shown in Fig. 5, melanocytes were transfected with p53-GFP lentiviral vectors and the transfection efficiency was monitored by GFP detection. qPCR analysis demonstrated that the relative levels of p53 and MMP9 were significantly increased in cells transfected with the p53-GFP lentiviral vector compared with those levels in the GFP vector control group, which was concomitant with decreases in TRPM1 and miR-211 levels (Fig. 5A). The effectiveness of the p53-GFP lentiviral vector was also confirmed by examining levels of the p53, TRPM1 and MMP9 proteins by western blotting analysis. The protein expression levels of p53 and MMP9 were significantly increased in cells transfected with the p53-GFP lentiviral vector compared with the control groups. Similar to the repression of miR-211 after transfection of the p53-lentiviral vector, the protein level of TRPM1 was also reduced compared with the control group. The results of immunofluorescence staining also revealed that the expression of MMP9 protein in transfected cells was increased (Fig. 5B). The Transwell assays showed that melanocyte migration was significantly increased in cells that had a lentiviral vector-mediated overexpression of p53 compared with the control group. Moreover, the overexpression of p53 induced a higher migration of melanocytes, which could be neutralized by GM6001 treatment (Fig. 5C). These data suggest that miR-211 exerts an anti-migration effect via the suppression of MMP9, whereas the overexpression of p53 in melanocytes directly reverses the inhibitory effect of miR-211 on cell migration. 


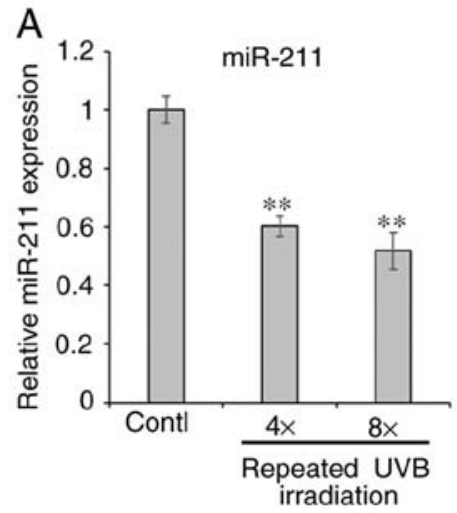

C
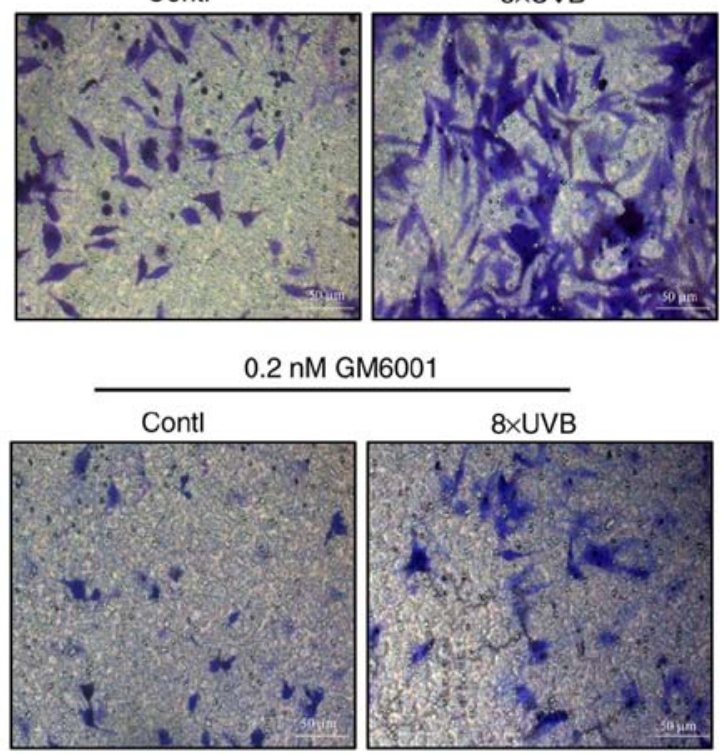

B

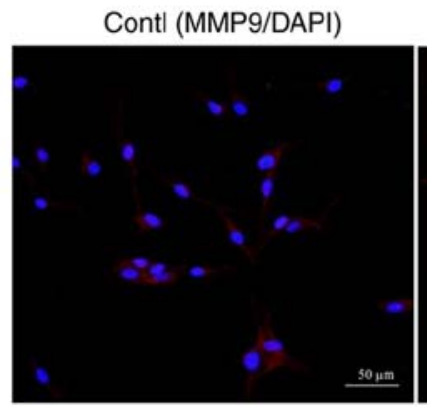

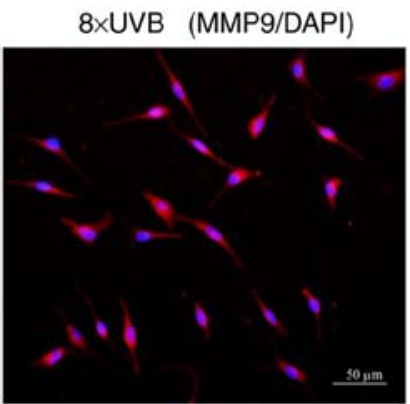

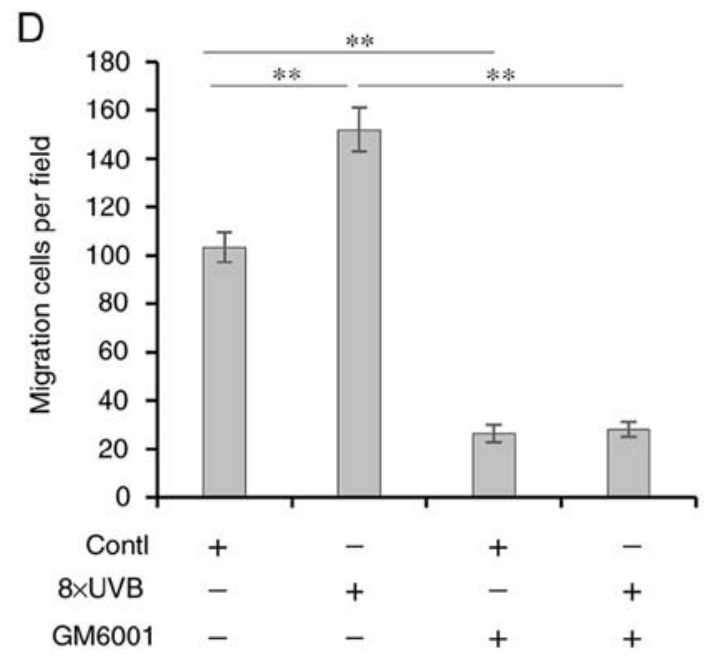

Figure 3. UVB-induced melanocyte migration elicited by suppression of miR-211 and the upregulation of MMP9. (A) Quantitative PCR was performed to measure the miR-211 levels of melanocytes treated with repeated UVB. Data represent the mean \pm standard deviation of three independent experiments. ${ }^{* *} \mathrm{P}<0.01$ vs. contl. (B) Melanocytes were immunostained with antibodies against MMP9 (red) and then counter-stained with DAPI to identify nuclei (blue). Representative fluorescence images of MMP9 merged with DAPI staining are shown. Scale bars: $50 \mu \mathrm{m}$. (C) Transwell cell culture chambers were used for cell migration assays. A total of $48 \mathrm{~h}$ later, migrating cells on the bottom surface of the inserts were stained with crystal violet and counted in five microscopic fields using a microscope. Representative images of migrating cells are shown. Scale bars: $50 \mu \mathrm{m}$. (D) Histogram showing the number of migrating cells in each Transwell as the mean \pm standard deviation of three independent experiments. ${ }^{* *} \mathrm{P}<0.01$ vs. contl or 8xUVB. UVB, ultraviolet B; ACTB, $\beta$-actin; MMP, matrix metalloproteinase; TRPM1, p53-transient receptor potential cation channel subfamily M member 1; miR, microRNA; DAPI, 4'6'-diamidino-2-phenylindole; contl, control.

\section{Discussion}

Although UVB-based phototherapy has been clinically proven to be an effective therapeutic option that improves repigmentation outcomes in vitiligo patients, the precise mechanism by which UVB activates and stimulates melanocyte migration in the skin is still unclear. The present study showed that p53 regulates MMP9 expression at the transcriptional level via a novel miR-211-mediated mechanism. Previous studies have revealed that the exons within the TRPM1 gene encode a $\mathrm{Ca}^{2+}$-permeable cation channel protein (melastatin) that is involved in regulating calcium homeostasis (21) and triggering melanosome transfer (22). However, the 6th intron of the TRPM1 gene also encodes miR-211, which acts as a critical modifier of melanin synthesis by targeting transforming growth factor- $\beta$ receptor 2 (23) and of cell migration by targeting MMP9 (24). MiR-211 has been predicted to target MMP9, which is abundantly expressed in normal melanocytes $(25,26)$.
Emerging evidence also indicates that p53 itself serves as a sensor of UVB-damage to control cell cycle arrest, DNA repair and apoptosis (27), constituting an extremely sensitive adaptive photoprotective response of the skin against UVB damage (28). There is a putative p53-binding motif that has been identified $25 \mathrm{~kb}$ upstream of the TRPM1 transcription start site (29). p53 is a transcription factor that binds to this site, leading to a downregulation in TRPM1 gene expression and a simultaneous decline in miR-211 expression, which as a result upregulates MMP9. In this study, it was found that in human melanocytes treated with a single dose or with repeated small doses of UVB, p53 expression levels were upregulated in a UVB dose-dependent manner. However, TRPM1 mRNA and protein levels were upregulated at lower UVB doses $(8.75$ or $\left.17.5 \mathrm{~mJ} / \mathrm{cm}^{2}\right)$ and were reduced at higher UVB doses (35 or $70 \mathrm{~mJ} / \mathrm{cm}^{2}$ ) of a single UVB radiation. In agreement with this observation, Devi et al (21) reported that endogenous p53 could be induced in melanocytes by UVB irradiation, whereas 

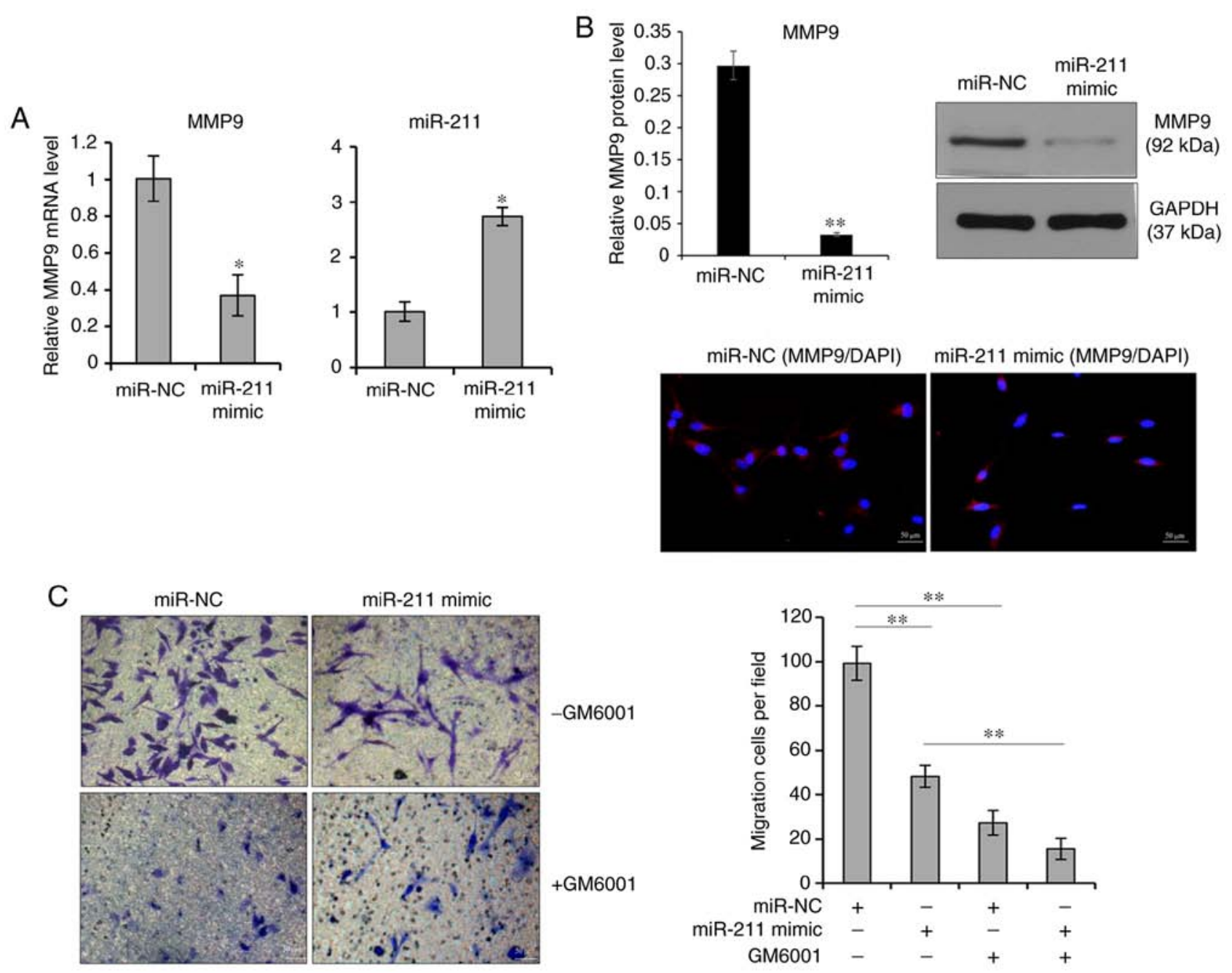

Figure 4. Effect of transient transfection of a miR-211 mimic on MMP9-mediated cell migration. (A) Melanocytes were transfected with the miR-211 mimic for $48 \mathrm{~h}$. Quantitative PCR was performed to measure the expression levels of miR-211 and MMP9 in cells transfected with the miR-211 mimic or the miR-NC. Data represent means \pm standard deviation of three independent experiments. ${ }^{*} \mathrm{P}<0.05$ vs. miR-NC. (B) Western blotting and immunofluorescence staining were carried out to examine changes of MMP9 protein levels in transfected cells. (C) Transwell cell culture chambers were used for cell migration assays. After being transfected with the miR-211 mimic or the miR-NC $48 \mathrm{~h}$, migrating cells on the bottom surface of each insert were stained with crystal violet and were counted in five microscopic fields using a microscope. Scale bars, $50 \mathrm{~mm} .{ }^{* *} \mathrm{P}<0.01 \mathrm{vs}$. miR-NC or miR-211 mimic. MMP, matrix metalloproteinase; TRPM1, p53-transient receptor potential cation channel subfamily M member 1; miR-NC, microRNA negative control.

exposure to $35 \mathrm{~mJ} / \mathrm{cm}^{2} \mathrm{UVB}$ also led to the downregulation of TRPM1 expression. Possible explanations for this observation are that: i) In response to small doses of UVB, melanocytes express higher protein levels of microphthalmia-associated transcription factor (MITF) in UVB-stressed melanocytes (30), the increased level of MITF would bind to the M-box in the upstream promoter region of the TRPM1 gene and activate its transcription $(23,31)$; ii) in response to repeated large doses of UVB, increased levels of p53 in UVB-damaged melanocytes inhibit TRPM1 gene expression. Therefore, the finely-tuned expression level of p53 and MITF in UVB-exposed melanocytes is critical for triggering MMP9-mediated cell migration.

The source of melanocytes that replenish the unpigmented skin in lesions vitiligo patients who have undergone marginal repigmentation may come from melanocytes existing at the border of the white macules $(2,5)$. It is, however, difficult to conceive how melanocytes can easily exit from a tightly interconnected epidermal microenvironment and enter a different location of the skin to re-establish a functional network with neighboring keratinocytes there. The present observations show that the MMP9 expression level is increased in a UVB dose-dependent manner as a result of the activation of the p53-TRPM1/miR-211-MMP9 axis. It is well documented that MMP9 is involved in the breakdown of the extracellular matrix and plays an important role in tissue remodeling during skin wound healing (32). In response to UVB irradiation, the UVB-stressed melanocytes activate p53, the activated p53 then binds DNA to downregulate TRPM1/miR-211 expression, which would lead to a transient increase of MMP9 and endow the stressed melanocytes with the ability to move out. Consistent with recent observations (33), melanocytes derived from vitiligo skin showed a very low level of MMP9, suggesting that the impaired expression of MMP9 exists in the perilesional skin of patients with vitiligo, which correlates with a poor response to UVB-based phototherapy. Future studies are necessary to examine the changes of all members of this axis in the three-dimensional epidermal models reconstructed from vitiligo melanocytes or/ normal melanocytes to further confirm the role of this axis in melanocyte migration induced by UVB-based phototherapy. 

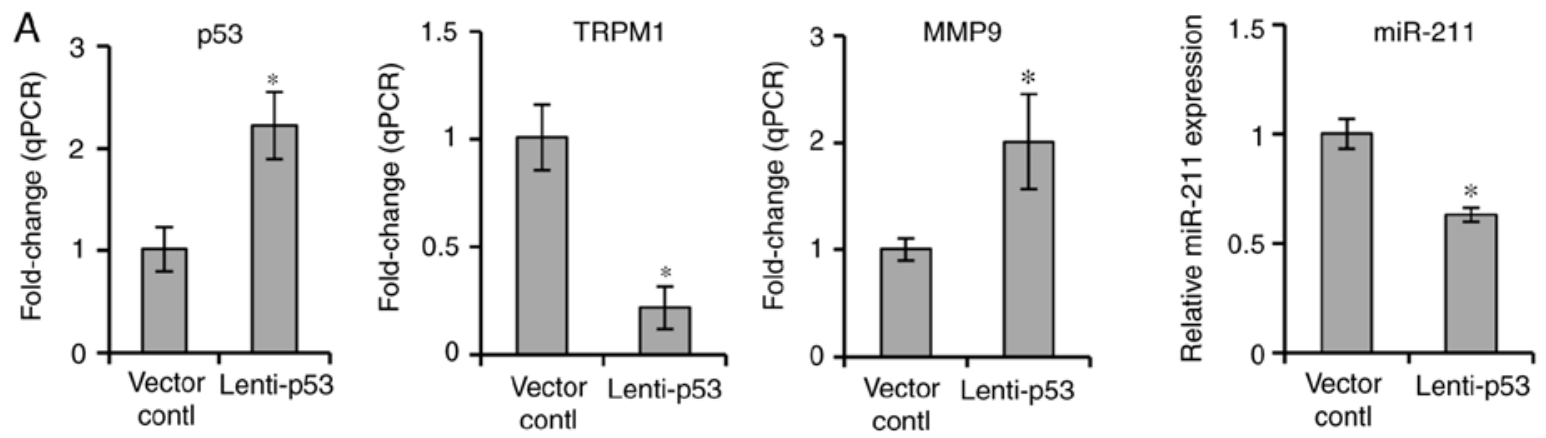

B Vector contl Lenti-p53

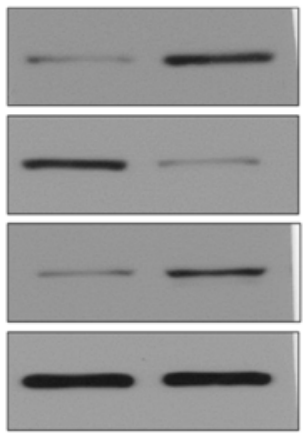

p53

$(53 \mathrm{kDa})$

TRPM1

$(182 \mathrm{kDa})$

MMP9

$(92 \mathrm{kDa})$

GAPDH

(37 kDa)
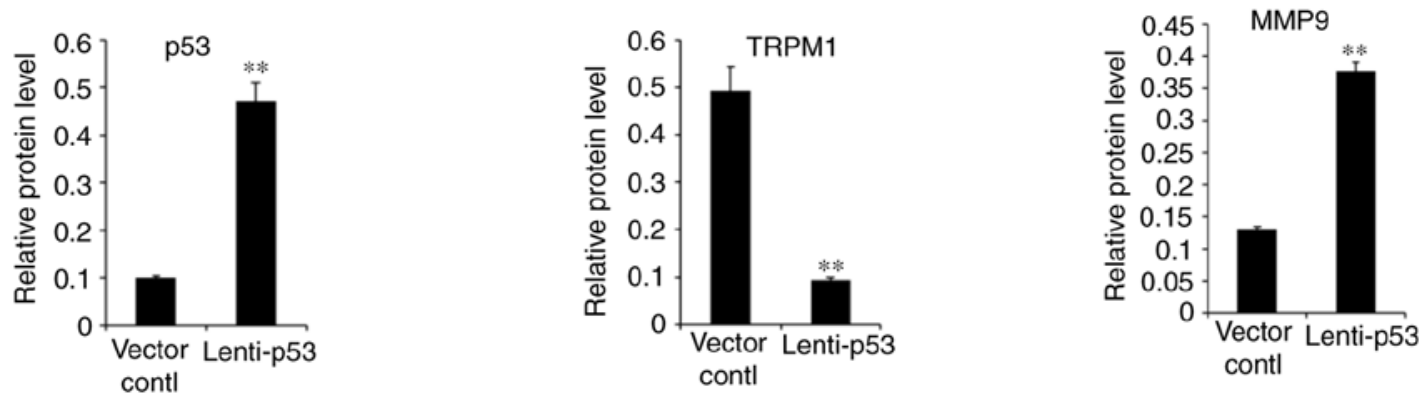

C
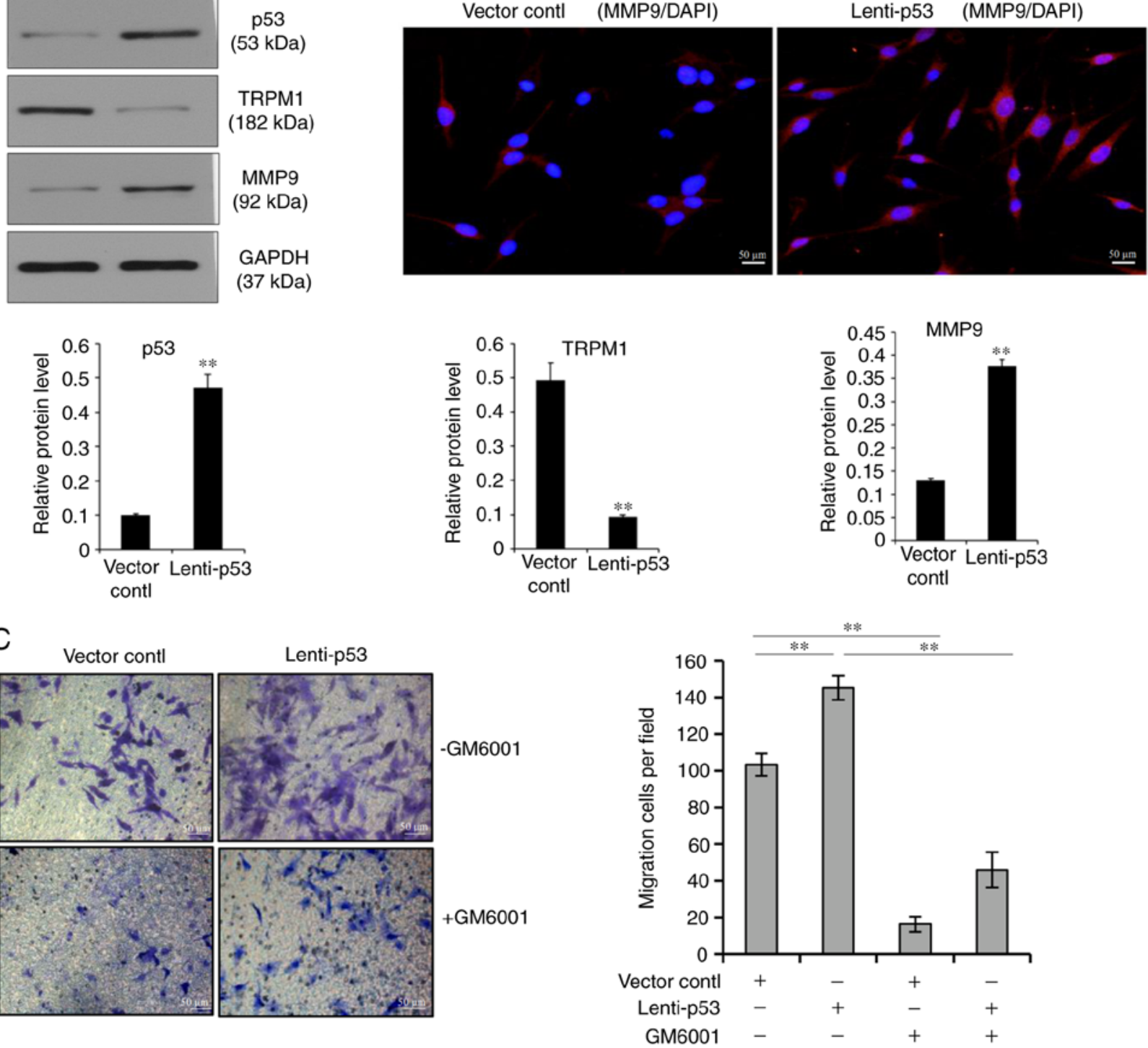

Figure 5. Effect of p53 overexpression on MMP9-mediated cell migration. (A) Melanocytes were transfected with p53-GFP lentiviral vectors, the transfection efficiency of the cells was monitored by GFP detection. Quantitative PCR analysis was performed to measure the p53, TRPM1, MMP9 and miR-211 level in melanocytes transfected with the p53-GFP lentiviral vector or with the GFP-vector control. Data represent the mean \pm standard deviation of three independent experiments. ${ }^{~} \mathrm{P}<0.05$ vs. vector control. (B) Western blot analysis was carried out to examine the protein levels of p53, TRPM1 and MMP9 in melanocytes transfected with the p53-GFP lentiviral vector or with the GFP-vector control. Immunofluorescence staining was carried out to examine changes of the MMP9 protein in transfected cells. (C) Transwell cell culture chambers were used for cell migration assays. Cells were transfected with the p53-GFP lentiviral vector or with the GFP-vector control. A total of $48 \mathrm{~h}$ later, migrating cells on the bottom surface of each insert were stained with crystal violet and counted in five microscopic fields using a microscope. Scale bars, $50 \mathrm{~mm} .{ }^{* *} \mathrm{P}<0.01$ vs. vector control or lenti-p53. MMP, matrix metalloproteinase; TRPM1, p53-transient receptor potential cation channel subfamily M member 1; GFP, green fluorescent protein; miR, microRNA.

Taken together, this is the first study that reveals the regulation of MMP9-mediated melanocyte migration via a novel mechanism driven by the p53-TRPM1/miR-211-MMP9 axis. Activation of this axis represents an attractive therapeutic 
target for improving repigmentation outcomes in vitiligo patients.

\section{Acknowledgements}

Not applicable.

\section{Funding}

The present study was supported by the National Natural Science Foundation of China (NSFC Grants 81573028).

\section{Availability of data and materials}

The data that support the findings of this study are available from the corresponding author upon reasonable request.

\section{Authors' contributions}

MS carried out the cell culture, cell transfection and wrote the paper. FM determined mRNA and protein levels by RT-PCR and western blotting. SJ, YS and LL carried out cell culture and migration assay. $\mathrm{XH}$ and JW collected skin samples and carried out the cell cultures. SX discussed the results, commented on the manuscript and analyzed the data. TCL designed the studies, analyzed the data and wrote the paper. All authors read and approved the final manuscript.

\section{Ethics approval and consent to participate}

The present study was approved by the Ethical Committee of the Renmin Hospital of Wuhan University. Written informed consent was obtained from each participant before enrollment.

\section{Patient consent for publication}

Not applicable.

\section{Competing interests}

The authors declare that they have no competing interests.

\section{References}

1. Gauthier Y, Cario Andre M and Taieb A: A critical appraisal of vitiligo etiologic theories. Is melanocyte loss a melanocytorrhagy? Pigment Cell Res 16: 322-332, 2003.

2. Goldstein NB, Koster MI, Hoaglin LG, Spoelstra NS, Kechris KJ, Robinson SE, Robinson WA, Roop DR, Norris DA and Birlea SA Narrow band ultraviolet B treatment for human vitiligo is associated with proliferation, migration, and differentiation of melanocyte precursors. J Invest Dermatol 135: 2068-2076, 2015.

3. Boniface K, Seneschal J, Picardo M and Taieb A: Vitiligo: Focus on clinical aspects, immunopathogenesis, and therapy. Clin Rev Allerg Immunol 54: 52-67, 2018.

4. Rashighi $M$ and Harris JE: Vitiligo pathogenesis and emerging treatments. Dermatol Clin 35: 257-265, 2017.

5. Birlea SA, Costin GE, Roop DR and Norris DA: Trends in regenerative medicine: Repigmentation in vitiligo through melanocyte stem cell mobilization. Med Res Rev 37: 907-935, 2017.

6. Cario M: DDR1 and DDR2 in skin. Cell Adh Migr 12: 386-393, 2018.

7. Wang JX, Fukunaga-Kalabis M and Herlyn M: Crosstalk in skin: Melanocytes, keratinocytes, stem cells, and melanoma. J Cell Commun Signal 10: 191-196, 2016.
8. Xiong XX, Ding GZ, Zhao WE, Li X, Ling YT, Sun L, Gong QL and Lu Y: Differences in the melanosome distribution within the epidermal melanin units and its association with the impairing background of leukoderma in vitiligo and halo nevi: A retrospective study. Arch Dermatol Res 309: 323-333, 2017.

9. O'Reilly-Pol T and Johnson SL: Melanocyte regeneration reveals mechanisms of adult stem cell regulation. Semin Cell Dev Biol 20: 117-124, 2009.

10. Esmat S, Mostafa W, Hegazy RA, Shalaby S, Sheth V, Youssef R and El-Mofty M: Phototherapy: The vitiligo management pillar. Clin Dermatol 34: 594-602, 2016.

11. Hirobe T and Enami H: Activation of melanoblasts and melanocytes after treatment with monochromatic excimer light and narrowband-ultraviolet B of skin of vitiligo patients. Int J Dermatol 58: 210-217, 2019.

12. Liu N, Matsumura H, Kato T, Ichinose $S$, Takada A, Namiki T, Asakawa K, Morinaga H, Mohri Y, De Arcangelis A, et al: Stem cell competition orchestrates skin homeostasis and ageing. Nature 568: 344-350, 2019.

13. Lei TC, Virador V, Yasumoto K, Vieira WD, Toyofuku K and Hearing VJ: Stimulation of melanoblast pigmentation by 8-methoxypsoralen: The involvement of microphthalmia-associated transcription factor, the protein kinase A signal pathway, and proteasome-mediated degradation. J Invest Dermatol 119: 1341-1349, 2002.

14. Chou WC, Takeo M, Rabbani P,Hu H,Lee W, Chung YR, Carucci J, Overbeek P and Ito M: Direct migration of follicular melanocyte stem cells to the epidermis after wounding or UVB irradiation is dependent on Mclr signaling. Nat Med 19: 924-929, 2013.

15. Coelho SG, Valencia JC, Yin L, Smuda C, Mahns A, Kolbe L, Miller SA, Beer JZ, Zhang G, Tuma PL and Hearing VJ: UV exposure modulates hemidesmosome plasticity, contributing to long-term pigmentation in human skin. J Pathol 236: 17-29, 2015.

16. Tariq H, Bella J, Jowitt TA, Holmes DF, Rouhi M, Nie Z, Baldock C, Garrod D and Tabernero L: Cadherin flexibility provides a key difference between desmosomes and adherens junctions. Proc Natl Acad Sci USA 112: 5395-5400, 2015.

17. Lei TC, Vieira WD and Hearing VJ: In vitro migration of melanoblasts requires matrix metalloproteinase-2: Implications to vitiligo therapy by photochemotherapy. Pigment Cell Res 15: 426-432, 2002

18. Yoshimura K, Tsukamoto K, Okazaki M, Virador VM, Lei TC, Suzuki Y, Uchida G, Kitano Y and Harii K: Effects of all- trans retinoic acid on melanogenesis in pigmented skin equivalents and monolayer culture of melanocytes. J Dermatol Sci 27 (Suppl.1): S68-S75, 2001.

19. Livak KJ and Schmittgen TD: Analysis of relative gene expression data using real-time quantitative PCR and the 2(-Delta Delta C(T)) method. Methods 25: 402-408, 2001.

20. Jiang G, Yang CS, Xu D, Sun C, Zheng JN, Lei TC and Liu YQ: Potent anti-tumour activity of a novel conditionally replicating adenovirus for melanoma via inhibition of migration and invasion. Br J Cancer 110: 2496-2505, 2014.

21. Devi S, Kedlaya R, Maddodi N, Bhat KM, Weber CS, Valdivia H and Setaluri V: Calcium homeostasis in human melanocytes: Role of transient receptor potential melastatin 1 (TRPM1) and its regulation by ultraviolet light. Am J Physiol Cell Physiol 297: C679-C687, 2009.

22. Hu QM, Yi WJ, Su MY, Jiang S, Xu SZ and Lei TC: Induction of retinal-dependent calcium influx in human melanocytes by UVA or UVB radiation contributes to the stimulation of melanosome transfer. Cell Prolif 50, 2017.

23. Dai XD, Rao CB, Li HR, Chen Y, Fan L, Geng HQ, Li S, Qu J and Hou L: Regulation of pigmentation by microRNAs: MITF-dependent microRNA-211 targets TGF- $\beta$ receptor 2 . Pigmemt Cell Melanoma Res 28: 217-222, 2015.

24. Margue C, Philippidou D, Reinsbach SE, Schmitt M, Behrmann I and Kreis S: New target genes of MITF-Induced microRNA-211 contribute to melanoma cell invasion. PLoS One 8: e73473, 2013.

25. Sahoo A, Lee B, Boniface K, Seneschal J, Sahoo SK, Seki T, Wang C, Das S, Han X, Steppie M, et al: MicroRNA-211 regulates oxidative phosphorylation and energy metabolism in human vitiligo. J Invest Dermatol 137: 1965-1974, 2017.

26. Asuthkar S, Velpula KK, Chetty C, Gorantla B and Rao JS: Epigenetic regulation of miRNA-211 by MMP-9 governs glioma cell apoptosis, chemosensitivity and radiosensitivity. Oncotarget 3: 1439-1454, 2012.

27. Cui R, Widlund HR, Feige E, Lin JY, Wilensky DL, Igras VE, D'Orazio J, Fung CY, Schanbacher CF, Granter SR and Fisher DE: Central role of p53 in the suntan response and pathologic hyperpigmentation. Cell 128: 853-864, 2007. 
28. Verschooten L, Declercq L and Garmyn M: Adaptive response of the skin to UVB damage: Role of the p53 protein. Int J Cosmet Sci 28: 1-7, 2006.

29. Wei CL, Wu Q, Vega VB, Chiu KP, Ng P, Zhang T, Shahab A, Yong $\mathrm{HC}, \mathrm{Fu} \mathrm{Y}$, Weng $\mathrm{Z}$, et al: A global map of p53 transcription-factor binding sites in the human genome. Cell 124: 207-219, 2006.

30. Terazawa $\mathrm{S}$ and Imokawa G: Signaling cascades activated by UVB in human melanocytes lead to the increased expression of melanocyte receptors, endothelin $\mathrm{B}$ receptor and c-KIT. Photochem Photobiol 94: 421-431, 2018.

31. Zhiqi S, Soltani MH, Bhat KM, Sangha N, Fang D, Hunter JJ and Setaluri V: Human melastatin 1 (TRPM1) is regulated by MITF and produces multiple polypeptide isoforms in melanocytes and melanoma. Melanoma Res 14: 509-516, 2004.
32. Baron J, Marquardt Y, Steiner T, Holzle F, Skazik-Voogt C, Heise R and Amann P: Effects of non-ablative fractional erbium glass laser treatment on gene regulation and skin physiology in human three-dimensional skin models. Laser Surg Med 48: 441-441, 2016.

33. Kumar R, Parsad D, Kanwar AJ and Kaul D: Altered levels of Ets-1 transcription factor and matrix metalloproteinases in melanocytes from patients with vitiligo. Br J Dermatol 165: 285-291, 2011

(i) (9) This work is licensed under a Creative Commons Attribution-NonCommercial-NoDerivatives 4.0 International (CC BY-NC-ND 4.0) License. 